\title{
Pediatric Acute Ischemic Stroke by Age-Group: A Systematic Review and Meta-Analysis of Published Studies and Hospitalization Records
}

\author{
Denise M. Oleske $^{a} \quad$ Xianbin Cheng $^{b}$ Anna Jeong ${ }^{c}$ Thomas J. Arndt ${ }^{d}$ \\ aGlobal Epidemiology, AbbVie, North Chicago, IL, USA; bepartment of Food Science and Human Nutrition, \\ University of Illinois, Urbana-Champaign, IL, USA; ${ }^{\complement}$ Neuroscience Clinical Development, AbbVie,

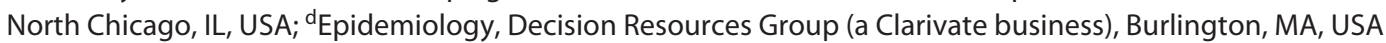

\section{Keywords}

Pediatric stroke - Neonatal stroke . Childhood stroke .

Epidemiology Systematic review

\begin{abstract}
Background: Although stroke is rare among the pediatric population, it is nevertheless associated with serious or lifethreatening consequences. The etiologic factors of acute ischemic stroke (AIS) are likely to vary over the course of childhood development. The incidence rates of AIS, not previously systematically examined by pediatric age subgroup, could guide studies of its etiology. Objective: The aim of this study is to evaluate the incidence rate of AIS by age-group in the pediatric population (aged $0-17 / 18$ years) and identify any common trends or sources of variability across different countries. Methods: Rates of pediatric AIS were collated from a systematic literature review of published studies globally (1983-2020) and hospitalization records from Europe and the USA (2015-2018). Records that were included in the analysis reported the code or description used for AIS diagnosis and age-specific data for children aged 0-17/18 years. AIS incidence rates were summarized by age-group, data source, country, and geographic region. A meta-analysis was conducted to assess the heterogeneity of AIS rates in
\end{abstract}

neonates. Results: The pooled AIS incidence rate was 5.6 per 100,000 children across all records. When only records reporting the AIS incidence rates for children across the full age range ( $0-17 / 18$ years) were analyzed, the pooled AIS incidence rate was 4.6 per 100,000 children and ranged from 7.0 per 100,000 (Germany) to 1.3 per 100,000 (Denmark). The highest pooled rates were observed in the 0-28-day agegroup (24.6 per 100,000 live births), declining to the lowest rates in the 5-9-year age-group, and rising again in the 1017/18-year age-group. AIS rates were the most heterogeneous in the 0-28-day age-group and across European countries. Significantly higher AIS rates in neonates were observed from hospital databases $(35.9$ per 100,000$)$ than in the literature (19.4 per 100,000). AIS rates may be underestimated as pediatric AIS events are rare and challenging to diagnose, and limited age-specific data are available. Conclusions: Incidence rates of pediatric AIS by age-groups followed a consistent overall pattern of a reverse J-shaped curve, with the highest rates in neonates, across predominantly European and North American countries. Further research is warranted to examine if this pattern is observed in other geographic regions and to identify AIS risk factors specific to different phases of childhood development.

(c) 2021 The Author(s).

Published by S. Karger AG, Basel karger@karger.com www.karger.com/ned

Karger $\stackrel{\text { ' }}{5}$

GOPEN ACCESS
(C) 2021 The Author(s)

Published by S. Karger AG, Basel

This is an Open Access article licensed under the Creative Commons Attribution-NonCommercial-4.0 International License (CC BY-NC) (http://www.karger.com/Services/OpenAccessLicense), applicable to the online version of the article only. Usage and distribution for commercial purposes requires written permission.
Correspondence to:

Denise M. Oleske, denise.oleske@ abbvie.com 


\section{Introduction}

Stroke is defined by the World Health Organization as "rapidly developing clinical signs of focal (at times global) disturbance of cerebral function lasting more than $24 \mathrm{~h}$ or leading to death with no apparent cause other than that of vascular origin" [1]. Ischemic stroke is a particular type of stroke caused by focal cerebral, spinal, or retinal infarction as a result of a sudden loss of blood flow to the brain and may lead to irreversible loss of neurological function, disability, and death [2-4]. Although stroke occurs at a higher rate in older adults, younger people are also affected. The global prevalence of ischemic stroke in the pediatric population was 40,000 in 2013 , representing an increase of approximately 35\% from 1990 [5], with mortality rates of $7-28 \%$ after childhood arterial ischemic stroke [6]. Childhood stroke survivors are at high risk of lifelong neurological deficits, including motor, language, cognitive, or behavioral deficits; and neurological sequelae, such as post-stroke epilepsy [7].

As the overall global prevalence of stroke is projected to increase over the next 30 years [8], investigation of the rates of acute ischemic stroke (AIS) in children may provide clues that could aid in stemming the rise. A Canadian study offers some evidence that clinical characteristics and predictors of arterial ischemic stroke differ between neonates and older children, indicating that age-appropriate treatments may be required [9]. However, pediatric AIS rates reported in the literature are inconsistent across studies and the rates for children of all ages from neonates to adolescents tend to be reported as a single group [10-12]. Despite this, no comprehensive analysis of the incidence of pediatric AIS by agegroups across multiple countries or regions exists. The purpose of this study was to determine whether the incidence of pediatric AIS varies by age-group and geographical region using both global published literature as well as hospitalization data for AIS in Europe and the USA.

\section{Materials and Methods}

\section{Study Design and Data Sources}

This descriptive cohort study integrated pediatric AIS data from a systematic literature review of global studies ("literature database") and hospital or medical claims records from France, Germany, Spain, UK, and the USA ("hospital database").

\section{Literature Database}

To identify articles that report pediatric AIS incidence rates, we searched the PubMed, MEDLINE, Scopus, and Google Scholar da- tabases through December 31, 2020, using the following search keywords or strings:

((Pediatric $[\mathrm{tw}]$ OR paediatric $[\mathrm{tw}]$ OR neonat*[tw] OR child*[tw] OR adolescent* $[\mathrm{tw}]$ OR infant [tw] OR perinatal[tw]) AND (stroke[mesh] OR "cerebral ischemia" [All Fields] OR "cerebral infarction" [All Fields])) AND (population-based[tw] OR hospital-based[tw] OR clinic[tw] OR register*[tw] OR registr*[tw] OR epidemiolog* $[\mathrm{tw}]$ ) AND (humans[mesh]) AND (Incidence[mesh:noexp] OR incidence[tiab] OR Prevalence[mesh:noexp] OR prevalence[tiab] OR recurren*[tw] OR relapse[tw] OR survival[tw]) AND ("2010"[Date - Entrez]: “3000”[Date - Entrez]) (“Stroke"[mesh] OR "cerebro vascular accident" [All Fields] OR "cerebral vascular accident"[All Fields] OR "CVA"[All Fields] OR "brain injury" [All Fields] OR "cerebral ischemia"[All Fields] OR "cerebral infarction" [All Fields]) AND (population-based[tw] OR hospital-based[tw] OR clinic[tw] OR register*[tw] OR registr* $[\mathrm{tw}]$ OR epidemiolog* $[\mathrm{tw}])$ AND (humans[mesh]) AND (Incidence[mesh:noexp] OR incidence[tiab] OR Prevalence[mesh:noexp] OR prevalence[tiab] OR recurren*[tw] OR relapse[tw] OR survival[tw]) AND (“2010"[Date - Entrez]: “3000”[Date - Entrez]).

A targeted literature search was also undertaken to identify all possible sources of data to best inform our analysis, and reference lists from the selected articles were reviewed to identify additional studies that met the above criteria but were not captured in the search. Publications were first screened by title and then abstracts to identify studies with AIS data in the pediatric population. All publications selected from the abstract screening phase were fully reviewed using the following criteria: (1) full article available in English; (2) published from 1983 onward; as more contemporary studies were expected to be more uniform in definitions of pediatric AIS, clinical assessments, and the use of imaging for diagnosis; (3) included $\geq 1$ pediatric age-group derived from a cohort or registry study; and (4) identified AIS cases had a clinical presentation consistent with stroke (i.e., neurological deficits, paresis, and seizures) and brain imaging consistent with AIS (if available); or used a diagnosis code consistent with AIS.

We extracted AIS rates computed from the number of events in an age-group and the corresponding sample size provided in the article or from governmental vital statistics for the study year and age-group. As the scope of this analysis was to identify incident cases of AIS, rates of recurrent strokes, other types of strokes that do not meet the above definition for AIS or AIS diagnostic coding, or strokes of unknown type were not included in the data analysis.

\section{Hospital Databases}

Country-specific hospital administrative claims databases for identifying cases of ischemic stroke as specified by International Classification of Diseases (ICDs)-10 or ICD-9 diagnosis coding were obtained (online suppl. Table 1; for all online suppl. material, see www.karger.com/doi/10.1159/000518281), with the assumption that all ischemic stroke events resulted in hospital admission. The analysis included patients who were admitted to hospital with an ischemic stroke event but then died.

We included the following hospital databases in this analysis due to the availability of large population-based pediatric samples and detailed diagnoses in these records:

1. The Health-care Cost and Utilization Project (HCUP) [13] is a US hospital discharge database that reports age-specific data on hospitalized ischemic events identified by ICD-10 codes, in- 
cluding those for neonates aged 0-28 days. This database uses a weighted sample of US community hospital discharges from various states to create a representative database of all US acute-care hospital discharges, includes both primary and alllisted diagnoses, and collects data from all noninstitutionalized payer systems.

2. The Hospital Episode Statistics (HES) [14] is a UK database that reports age-specific data on hospitalized AIS events identified by ICD-10 codes to the first decimal place and includes both primary and all-listed diagnoses and collects data from all National Health Service Commissioning Group acute-care hospitals.

3. Direction de la recherche, des études, de l'évaluation et des statistiques (DREES) [15] is a French hospital discharge database that reports primary diagnosis events by ICD-10 codes from all acute-care hospital discharges for stays $\geq 24 \mathrm{~h}$ for medical, surgical, obstetrics/gynecological, and dental care. Because DREES groups together all ICD-10 codes relating to conditions of the perinatal period (P00-P96), we generated a ratio for P91.0 (perinatal cerebral ischemia) to P00-P96 from HES 2018 [14] and applied this to DREES. We also adjusted the primary (main) diagnosis events to all listed diagnosis events, using a ratio of primary diagnoses to all listed diagnoses from HES 2018 [14]. We used a Danish study [16] that reported the case counts by $<1$ year, $0-<28$ days, and $\geq 28$ days $-<12$ months, to derive ratios to stratify the events in the " $<1$ year" age-group into the following key age-groups of interest: " $0-<28$ days" and " $\geq 28$ days $-<12$ months."

4. The German Federal Statistical Office database [17] reports age-specific data on hospitalized patients by primary diagnosis and ICD-10 codes to the first decimal place from all public and private community hospitals within German federal territories.

5. The Conjunto Mínimo Básico de Datos - Hospitalización [18] is a Spanish hospital discharge database with age-specific, alllisted AIS events by ICD-9 codes. Because there is no comparable ICD-9 code for P91.0 (neonatal cerebral ischemia), we used the ratio of P91.0 to all ICD-10 stroke codes from HES [14] and applied it to the Conjunto Mínimo Básico de Datos - Hospitalización data for all ICD-9 stroke codes for that age-group. To isolate incident AIS events from all stroke events, we used ratios derived from the international Vascular Effects of Infection in Pediatric Stroke study [19] to remove recurrent stroke events from the hospital discharge data. Where the age-groups reported did not match the desired age-groups for this study, we used a weighted average of $8.6,10.8,15.7$, and $10.3 \%$ for non-neonates aged 28 days -1 year, $1-4,5-9$, and $10-17 / 18$ years, respectively [20]. For neonates, we used a stroke recurrence rate of $0.68 \%$ derived from multiplying a hazard ratio of 0.06 for neonatal versus non-neonatal stroke recurrence reported in a US study [21] and the average stroke recurrence rate among non-neonates. To ensure the reliability of the AIS rate estimates obtained from the hospital databases, we confirmed the selection of diagnosis codes, correct application of all formulas to the data source, and accurate data entry of computed rate numbers. All data used by Decision Resources Group in this analysis were previously de-identified by its source and therefore do not fall under Protected Health Information.

\section{Data Extraction}

Data were extracted from the literature studies and from the hospital discharge databases and integrated into a master database.
Any data record from a published study or a hospital discharge database was included if it satisfied all of the following criteria: (1) AIS or ischemic stroke diagnosis cited in the publication or AIS diagnosis code cited for a hospital discharge record; (2) incidence data for any of the age-groups from age 0 days ( $\geq 28$ weeks of gestation) to 17 years (or to 18 years for US records); and (3) for a published study, the incidence rate reported for at least one of the age-groups, or the numerator and the denominator for any of the age-groups from which the incidence rate could be calculated. Data from published studies that could not be abstracted to provide an age-group that aligned with our predefined groups (e.g., studies with an age subgroup of 15-21 years), did not report either the numerator or denominator for an age-group, or could not be used to determine incident stroke number or rate in an age-group, were excluded.

\section{Assessment of Risk of Bias}

The risk of bias within studies is ascertained from the study design and the data source. The quality of a published study was rated high if all clinical and imaging criteria were specified; medium, if only clinical criteria were specified; and low if only the diagnosis or diagnosis coding was provided. To reduce bias, we excluded any records that lacked granularity regarding the AIS diagnosis or age-groups. There is no known published measure to assess the risk of bias across published studies with data directly from multiple hospital databases. However, as the majority of publications are based on hospital data, these records should be broadly comparable in that children experiencing acute signs and symptoms are likely to seek emergency hospital care.

\section{Outcomes}

The primary outcome was the incidence rates of pediatric AIS in the following age-groups: 0-28 days, 29 days-<1 year, 1-4, 5-9, and $10-17$ years (or $10-18$ years in US patients). Pediatric AIS was defined as the first ischemic stroke that occurred in children aged 0 days to 17 years (or 18 years for US records). The incidence rate was reported per 100,000 live births for those aged 0-28 days and per 100,000 children for those aged 29 days or older. Secondary outcomes included the overall and/or age-group incidence rates of AIS by country or geographical region and by data source (hospital database or literature). A meta-analysis was conducted to explore the variability of AIS incidence in neonates (aged 0-28 days).

\section{Statistical Analysis}

Statistical analyses were conducted in $\mathrm{R}$ (version 3.6.0) on the Red Hat Linux system (64-bit) with the following packages: tidyverse (version 1.3.0) and metaviz (version 0.3.1). A graphic user interface was developed using R Shiny (version 1.4.0.2), the package shinydashboard (version 0.7.1), and kableExtra (version 1.1.0). All programming codes for the data summarization, visualization, and statistical analyses are provided in the online supplementary material.

\section{Calculation of AIS Incidence Rates}

Incidence rates were abstracted or calculated for the overall pediatric population and each of the age-groups for individual data records. 95\% confidence intervals (CIs) were calculated using the Poisson method with both the numerator (number of ischemic stroke events) and the denominator (population in a specific age- 


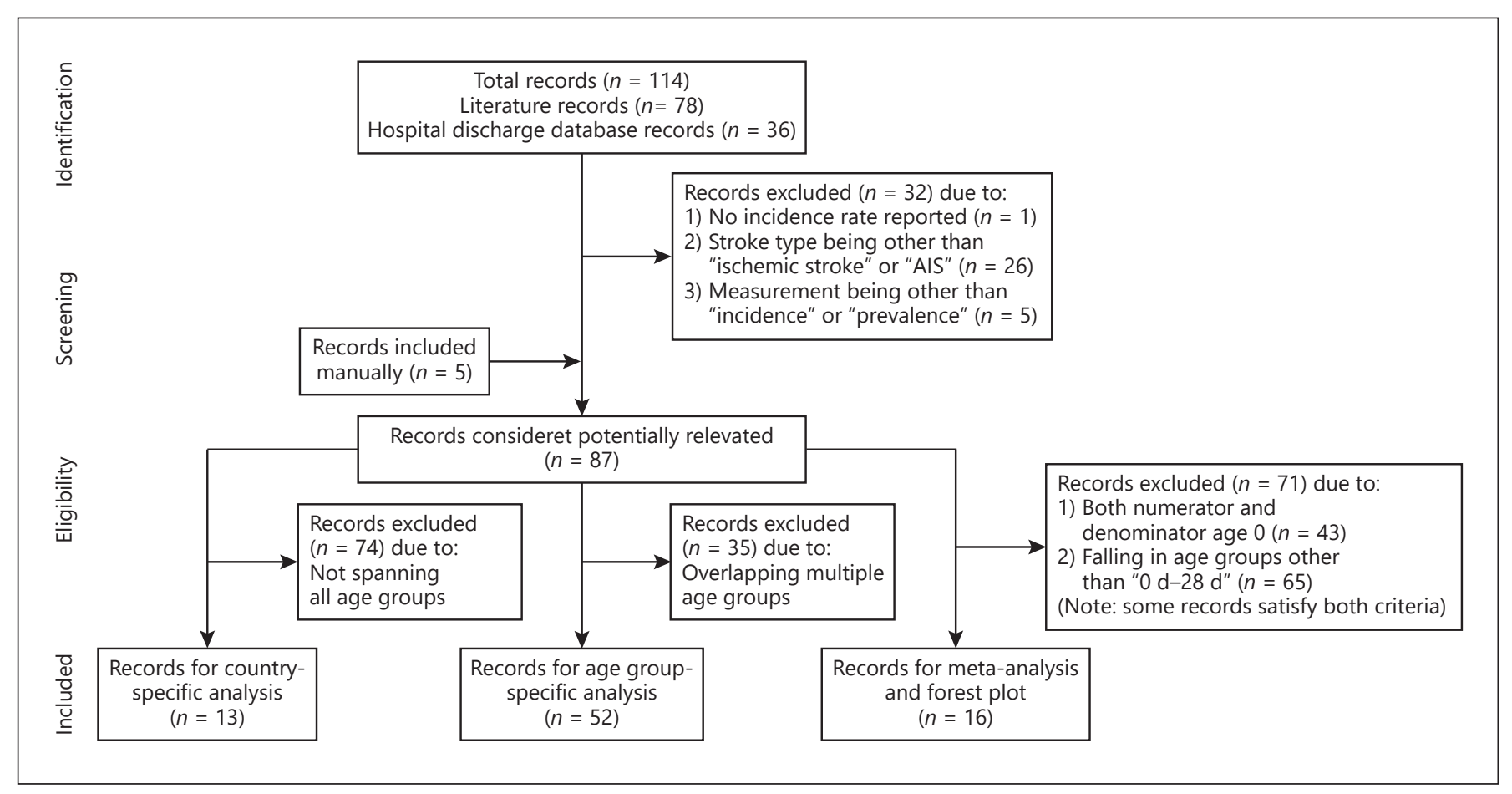

Fig. 1. Flowchart of study selection (adapted from PRISMA: available from www.prisma-statement.org). AIS, acute ischemic stroke; $d$, days.

group) from each study; or when the denominator was not available, by

$$
\frac{\text { numerator } \times 100,000}{\text { incidence rate }}
$$

[22]. Mean incidence rates (95\% CI) were calculated based on pooled data by country and age-groups and were assumed to follow a normal distribution.

\section{Meta-Analysis of Variability}

The formulas used in this meta-analysis were adapted from Neyeloff et al. [23]. In brief, we calculated the standard error (SE) of each data record using the reported incidence rate and denominator. SEs and incidence rates were used to produce the forest plots using the R package "metaviz." Heterogeneity was reported using the $I^{2}$ statistic, calculated from Cochran's Q statistic based on the reported incidence rates and weight (inverse of the SE squared) of each data record included in the analysis.

\section{Results}

\section{Selection of Records}

The initial literature search identified 10,893 potential articles for inclusion, 30 of which fulfilled the criteria for inclusion in the combined database. A total of 114 records were included in the combined database: 78 records from the 30 published studies and 36 hospital records (Fig. 1). Additional screening identified the final 87 records presented in this analysis, which comprised 57 records from 26 published studies and 30 hospital records (online suppl. Table 2).

The published studies included in this analysis were predominantly retrospective reviews of medical records or from registries where data are collected after the AIS event occurred. It is not feasible to conduct prospective epidemiological studies in such a broad age range of children due to the rarity of pediatric AIS. The majority of the data was originated from hospital records. There is no recall bias as the diagnoses of patients admitted to the hospital are coded directly from the hospital notes. However, AIS rates may be underestimated in hospital data owing to the difficulty in determining pediatric stroke in the youngest age-groups. All of the records included in the analysis were from Europe or North America, except for 1 study from Asia (Taiwan).

\section{Overall AIS Rates}

Overall, the pooled AIS incidence rate across all records was 5.61 per 100,000 children. However, this was reduced to 4.58 per 100,000 children when only records 

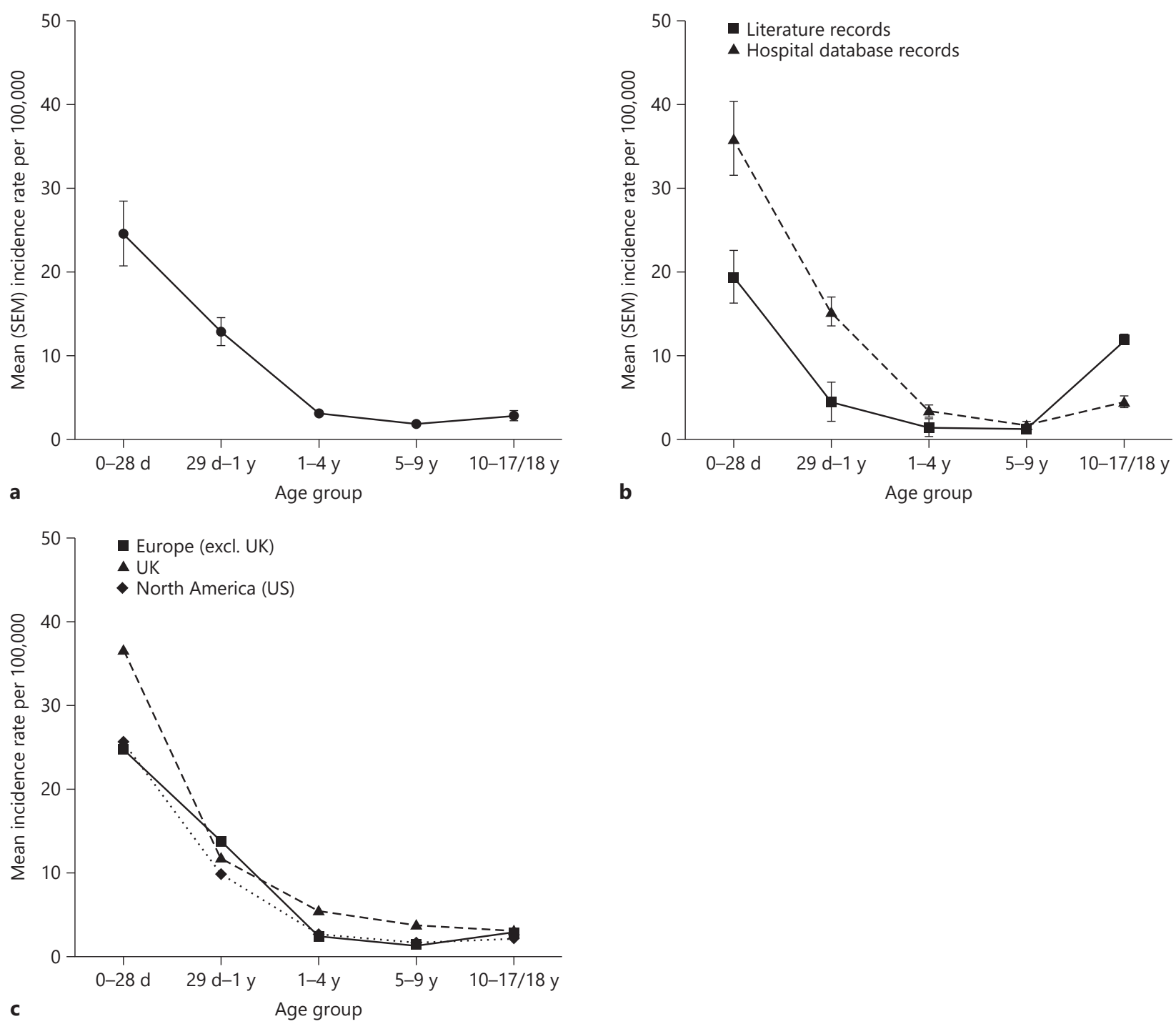

Fig. 2. Incidence rates of pediatric AIS by age-group per 100,000 children (per 100,000 live births for 0-28 day age-group), 1992-2018. a Overall. b By data source. c By geographic region/country (only for countries which reported separate incidence rates for children across each of the 5 age subgroups). d, days; SEM, standard error of the mean; y, years; AIS, acute ischemic stroke.

that reported the full age range ( $0-17 / 18$ years) were included in the analysis. The overall AIS rates grouped by hospital databases (5.97 per 100,000 children) and by the literature studies (5.17 per 100,000 children) were comparable.

\section{AIS Rates by Age-Group}

From 52 records pooled across all data sources, the incidence rates of AIS by age-group followed a reverse Jshaped pattern (Fig. 2a; online suppl. Table 3). The age
0-28 day group was associated with the highest incidence rates of AIS (24.55 per 100,000 live births). AIS incidence rates declined with increasing age, until reaching a trough at the 5-9-year age-group, before a slight increase in the age 10-17/18-year age-group.

This reverse J-shaped pattern was consistently observed in age-group analyses across both records from hospital databases or the published literature (Fig. 2b; online suppl. Table 4) and across regions or countries for which the full range of age-groups are available (Fig. 2c; 
Fig. 3. Incidence rates of pediatric AIS per 100,000 children, overall ages 0 days to 17 years by country*, 1984-2018. (US data include ages up to and including 18 years.) * Only included records which reported incidence rates for children across all agegroups (0-17/18 years). AIS, acute ischemic stroke.

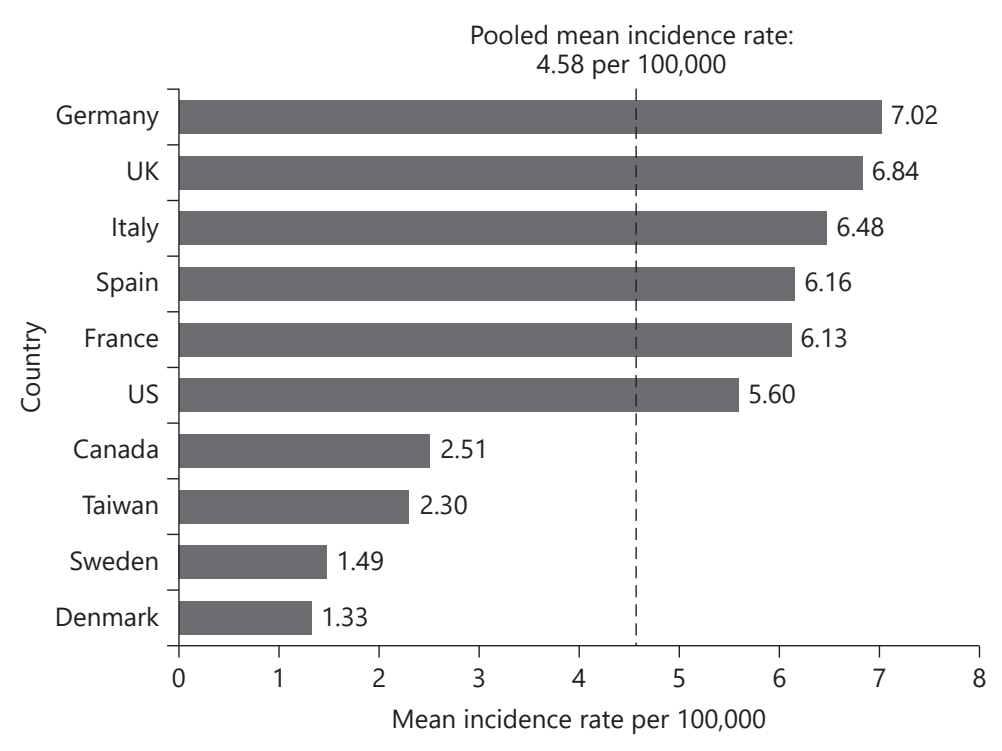

8). The incidence rates of neonatal AIS were highly variable across studies $\left(I^{2}=98.33 \%\right.$; Fig. $\left.4 a\right)$. We did not observe any trends by publication/database year. While US records were relatively consistent $\left(I^{2}=39.56 \%\right)$, there was high variability across records from European countries, excluding UK ( $I^{2}=98.63 \%$; online suppl. Table 8$)$.

To determine if the variability was due to the different sources of data used, the rates derived from the 5 hospital databases compared to those from the 11 studies were examined using meta-analysis. Although the variability was high in both groups (hospital databases: $I^{2}=96.06$; literature studies: $I^{2}=95.71$ ), the pooled estimate of the neonatal AIS rate derived from the model was significantly higher from the hospital databases (29.26 [95\% CI 27.95, 30.57] per 100,000 live births) than that from the literature studies (10.64 [95\% CI 9.85, 11.44] per 100,000 live births; Fig. 4b).

\section{Discussion}

\section{Summary of Findings}

To the authors' knowledge, this is the first epidemiological study to examine the rate of childhood AIS by age subgroups across multiple countries. Based on a systematic review of published studies and hospital records, we demonstrated that the incidence rates of pediatric AIS followed a reverse J-shaped pattern, with the highest rates 


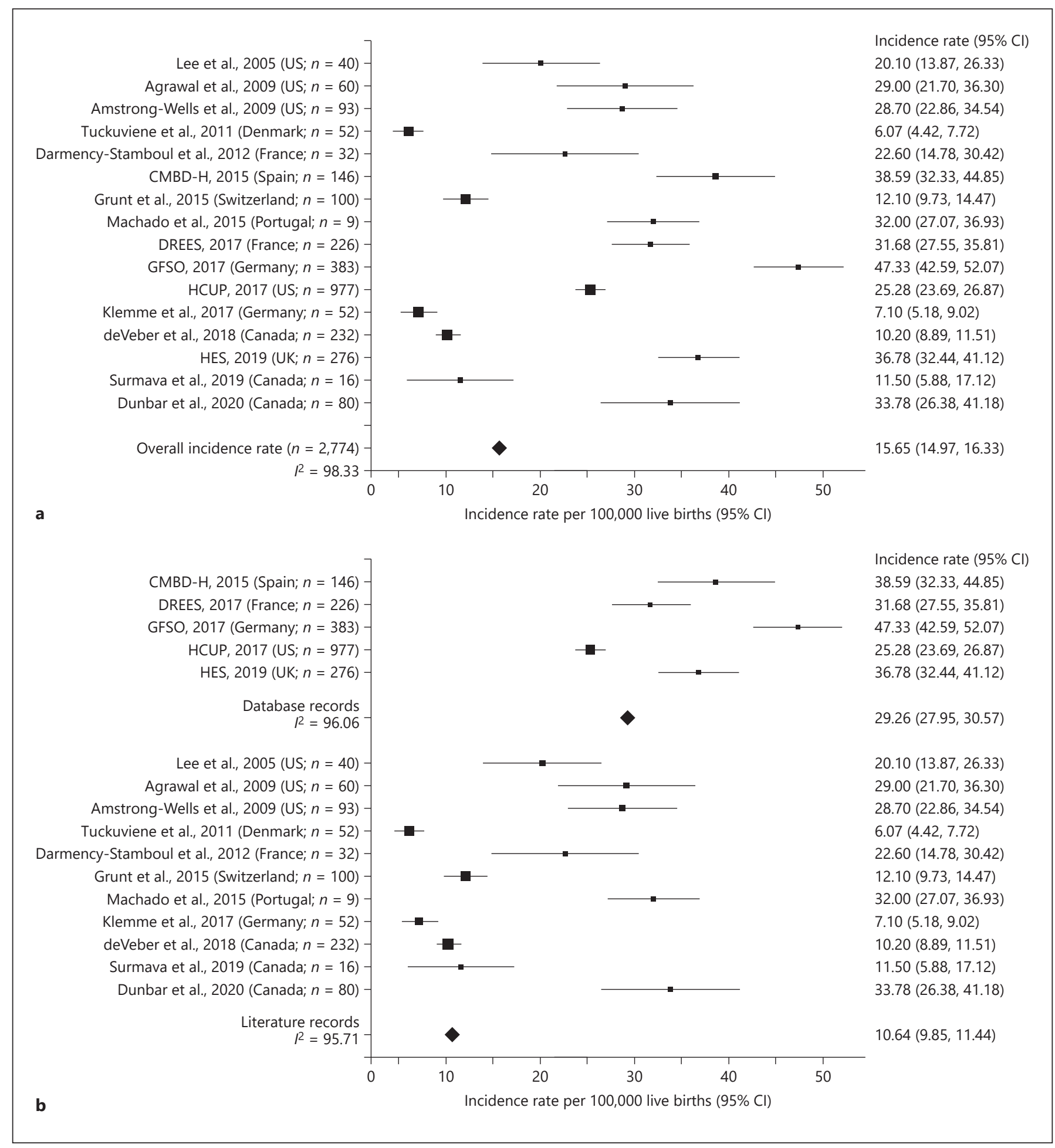

Fig. 4. Incidence rates of pediatric AIS per 100,000 live births, ages 0-28 days, 1992-2018. a Overall. b By data source. CI, confidence interval; CMBD-H, Conjunto Mínimo Básico de Datos - Hospitalización; DREES, Direction de la recherche, des études, de l'évaluation et des statistiques; GFSO, German Federal Statistical Office; HCUP, Health-care Cost and Utilization Project; HES, Hospital Episode Statistics; AIS, acute ischemic stroke. 
of AIS observed in the 0-28 days age-group, decreasing to a trough in the 5-9-year age-group, and rising again in the 10-17/18-year age-group. This pattern was observed consistently across data sources and the geographic regions examined.

\section{Comparison with Other Studies}

While a Canadian study has previously shown that higher rates of AIS were observed among neonates than older children [9], our study showed for the first time that this trend extends to other studies across various countries. We found a higher pooled rate of AIS in neonates aged 0-28 days (24.6 per 100,000 live births) than that reported for this age-group in other studies $[9,24]$. This may be in part explained by the inclusion of hospital data. Additionally, neonates exhibited the greatest variability in AIS incidence rate out of all the age-groups analyzed. The greatest variability was observed within Europe, with AIS rates ranging from 6.1 (Denmark) - 47.3 (Germany) per 100,000 live births, while limited variability was observed in the USA (20.1-29.0 per 100,000 live births).

The pooled annual incidence rates of AIS in the overall age-group ( 4.58 per 100,000 children aged $0-17 / 18$ years) reported in this analysis were within the range for those reported in prior studies (0.6-7.9 per 100,000 children) $[9,16,25,26]$. However, to the authors' knowledge, this is the most comprehensive global epidemiological study to examine pediatric AIS incidence by multiple agegroups and geographic region to date. Assuming all incident AIS cases are admitted to hospital enhances the possibility of a correct diagnosis in a timely manner, hospital data can provide a useful benchmark or sensitivity analysis for published studies and are generally representative of the AIS incidence rates in the geographic region from which cases are admitted. Previous studies were largely restricted in geographic representation or provided data from specialty centers, and publications based on hospitalization data only reported AIS rates of the overall agegroup (0-18/19 years) [11].

\section{High Variability for Pediatric AIS Rates}

While the overall trends in pediatric AIS rates by agegroup were generally consistent, there was a large variation in absolute incidence rates across records. This may be partially explained by the data source, as higher rates were reported in hospital databases than in the literature. Possible reasons for this may be that the hospital databases used in this analysis included a larger population with higher granularity in terms of age-groups and were more contemporary (2015-2018) than that of the published literature (1992 onwards), which may be associated with better identification of pediatric AIS. Another source of variability may be the difference in the definition of AIS between records and over time. However, we have attempted to control for this by using clearly defined criteria in selecting records for inclusion. Due to the high AIS rates observed in neonates, overall AIS rates may also be underestimated if neonates were excluded from the analysis.

Country-specific variability may also be attributed to structural differences such as case ascertainment associated with retrospective studies [27], whether the neonatal age-group was included or not, as well as other factors including etiologic differences of AIS and pre- and postnatal health-care services. Regardless, due to potential differences in patient populations and study designs, the potential variability of country-specific AIS rates should be interpreted with caution.

\section{Possible Etiologic Factors for Neonatal AIS}

Risk factors associated with neonatal AIS, including biologic or environmental factors pertaining to the mother, her pregnancy, the delivery of the newborn, or neonatal comorbidities may account for the high and variable rates of AIS during the neonatal period [24, 28]. Specific maternal and pregnancy-related factors that have been associated with neonatal AIS include, but are not limited to, pre-eclampsia, prolonged rupture of membranes, and infection [29]. Risk factors specific to the neonate include hereditary thrombophilia, coagulation disorders, and cardiac lesions [30].

\section{Possible Etiologic Factors for AIS in Older Children}

The etiologies of childhood and adolescent stroke are diverse and include, but are not limited to, cardiac causes, arteriopathies (intracranial or extracranial), vasculitis and autoimmune disorders (i.e., systemic lupus erythematosus), hematologic disorders (i.e., sickle cell disease), and traumatic injury [30-32]. Of note, recent head or neck trauma has been identified as a significant risk factor for childhood AIS across all age-groups, with children who experienced more severe trauma reflected by loss of consciousness or hospital admission at a higher risk [33]. Children may also be susceptible to internal carotid dissection following trauma of the soft palate by objects such as pencils $[32,34]$.

Adolescent AIS encompasses both pediatric and adult clinical features [31]. While the relatively favorable prognosis and low recurrence rate (with the exception of pa- 
tients with vasculopathy) observed in adolescent AIS are common features of pediatric AIS, the etiology, risk factors, and presenting symptoms of adolescent AIS are more similar to that of adult AIS.

Therefore, the slight increase in AIS rates in adolescents may reflect a shift toward increased risk factors more commonly associated with young adults, such as obesity, hypertension, diabetes, lipid disorders, and tobacco use, which are more common in adolescents than young children, and have been identified as coexisting conditions in adolescents and young adults who experience AIS [35-37]. Adolescents are also more likely to use prescription or illegal drugs, which may increase the risk of cerebral infarcts and/or thrombosis [32]. Moyamoya disease, an occlusive cerebrovascular disease, is another important cause of pediatric stroke. The onset of moyamoya disease peaks at around 10 years of age in children (and at 40 years of age in adults), potentially leading to an increased risk of stroke in children in this age-group [38, 39].

\section{Strengths and Weaknesses}

The rarity of pediatric AIS and the population affected renders a traditional longitudinal epidemiological study infeasible. Although active surveillance of cases may be a suitable alternative, this is a resource-intensive approach and may have limited generalizability. The use of hospital data to estimate the magnitude of this rare disease strengthens knowledge of the magnitude of the incidence rates of pediatric ischemic stroke by age-group from published studies which are largely based upon small samples from specialty centers. This aided in obtaining high $I^{2}$ values in the subgroup analyses of neonatal stroke. The software developed for this study is available as an online suppl. file and should facilitate future studies of this rare condition as it enables a link between the data elements and the visualization of their results.

There were several limitations of this analysis. First, the diagnostic criteria for AIS were not uniform across all studies and datasets. The diagnosis of AIS in the youngest age-group (0-28 days) is the most challenging and may not be accurately be determined at birth. Nevertheless, we observed a consistent overall pattern by age-group based on the combined hospital datasets and published studies. Second, the high level of age and diagnosis granularity required for this analysis limited the number and geographical regions of the studies and/or databases included. As the data included originated only from countries considered as high-income nations according to the World Bank classification [40], and pre- dominantly from European and North American countries, it is unclear whether these results can be generalized to other geographical regions and low-income nations. Third, this analysis was not adjusted for study dates or periods. Although only studies from 1983 onward were included, given the increase in pediatric AIS incidence between 1990 and 2013 [5], AIS rates may be underestimated in older studies or those in which data were collected over long periods and may have contributed to the heterogeneity in AIS rates observed between studies.

\section{Conclusion and Future Perspectives}

Even though we found some methodological differences in how pediatric AIS rates were reported across studies and databases, pediatric AIS rates follow a consistent reverse J-curve pattern by age-group across predominantly European and North American countries. These results suggest that the etiology of pediatric AIS may vary by age, and support future research, such as determining the appropriate sample sizes and identifying age-appropriate treatments. The consistency in this pattern across geographical regions studied suggests common etiological factor or factor groupings occurring within different developmental stages in childhood. This needs to be examined in studies of other nations to determine if the same pattern emerges to develop hypotheses if environmental, social, or other factors should be explored. The trending increase in the AIS incidence at the later ages of childhood may suggest a window of time for targeting preventive initiatives after future studies obtain more information about risk factors during this time period. Last, for the clinicians, an awareness of the age-dependent variation in AIS rates demonstrated in this analysis may also facilitate diagnosis, while policy makers may use these data as a baseline to monitor trends for any population-based interventions.

\section{Acknowledgments}

The authors would like to thank the following individuals from Decision Resources Group: Alison Isherwood, PhD, for project management and Stephanie Niquita Phankon, MBBS, MPH and Deepti Sharma, BDS, MPH, for assistance with literature sourcing. The authors would also like to thank Kurt White, $\mathrm{PhD}$, formerly with Decision Resources Group, for supporting the hospital database analysis. Editorial support was provided by Hilary Wong, $\mathrm{PhD}$, of 2 the Nth (Cheshire, UK). 


\section{Statement of Ethics}

The International Society of Pharmacoepidemiology (ISPE) Guidelines for Good Pharmacoepidemiology Practice (2015) were followed in the conduct of this study. All hospital discharge data analyzed as part of this work are de-identified with no personal identifiers.

\section{Conflict of Interest Statement}

Drs. Oleske and Jeong are full-time employees of AbbVie, Inc. and may own stock or stock options, and a final report on pediatric ischemic stroke in the USA and the EU5. Mr. Arndt is a fulltime employee of Decision Resources Group. Dr. Cheng was formerly a graduate student contractor for AbbVie and performed the data extraction and analysis for the project.

\section{Funding Sources}

This work was supported by AbbVie. AbbVie provided funding to Decision Resources Group, a Clarivate business, to produce a hospitalization analysis and also funding to 2 the Nth (Cheshire, UK) for editorial support.

\section{Author Contributions}

Conceptualization was contributed by X.C., D.M.O., and A.J. Data curation was done by X.C., D.M.O., and T.J.A. Formal analysis was performed by X.C. and D.M.O. Methodology was contributed by X.C., D.M.O., A.J., and T.J.A. Software was given by X.C. Supervision was done by D.M.O. Writing original draft was contributed by D.M.O. and X.C. Writing, review and editing was done by D.M.O., X.C., A.J., and T.J.A.

\section{Data Availability Statement}

The authors confirm that, with the exception of data from the Healthcare Cost and Utilization Project (HCUP), all data are freely and publicly available as links within the article and/or its supplementary materials. HCUP is published by the Agency for Healthcare Research and Quality (a part of the US Department of Health and Human Services) and can be purchased for a one-time fee here: https://www.hcup-us.ahrq.gov/.

\section{References}

1 Aho K, Harmsen P, Hatano S, Marquardsen J, Smirnov VE, Strasser T. Cerebrovascular disease in the community: results of a WHO collaborative study. Bull World Health Organ. 1980;58:113-30

2 GBD 2016 Stroke Collaborators. Global, regional, and national burden of stroke, 19902016: a systematic analysis for the Global Burden of Disease Study 2016. Lancet Neurol. 2019;18:439-58.

3 Phipps MS, Cronin CA. Management of acute ischemic stroke. BMJ. 2020;368.

4 Sacco RL, Kasner SE, Broderick JP, Caplan LR, Connors JJ, Culebras A, et al. An updated definition of stroke for the 21st century: a statement for healthcare professionals from the American Heart Association/American Stroke Association. Stroke. 2013;44:2064-89.

5 Krishnamurthi RV, deVeber G, Feigin VL, Barker-Collo S, Fullerton H, Mackay MT, et al. Stroke prevalence, mortality and disabilityadjusted life years in children and youth aged 0-19 years: data from the Global and Regional Burden of Stroke 2013. Neuroepidemiology. 2015;45:177-89.

6 Greenham M, Gordon A, Anderson V, Mackay MT. Outcome in childhood stroke. Stroke. 2016;47(4):1159-64.

7 deVeber GA, MacGregor D, Curtis R, Mayank S. Neurologic outcome in survivors of childhood arterial ischemic stroke and sinovenous thrombosis. J Child Neurol. 2000;15: $316-24$.
8 Wafa HA, Wolfe CDA, Emmett E, Roth GA, Johnson CO, Wang Y. Burden of stroke in Europe: thirty-year projections of incidence, prevalence, deaths, and disability-adjusted life years. Stroke. 2020;51:2418-27.

9 deVeber GA, Kirton A, Booth FA, Yager JY, Wirrell EC, Wood E, et al. Epidemiology and outcomes of arterial ischemic stroke in children: the Canadian Pediatric Ischemic Stroke Registry. Pediatr Neurol. 2017;69:58-70.

10 Chung B, Wong V. Pediatric stroke among Hong Kong Chinese subjects. Pediatrics. 2004;114:e206-12.

11 Lo W, Stephens J, Fernandez S. Pediatric stroke in the United States and the impact of risk factors. J Child Neurol. 2009;24:194-203.

12 Surmava AM, Maclagan LC, Khan F, Kapral MK, Hall RE, deVeber G. Incidence and current treatment gaps in pediatric stroke and TIA: an Ontario-wide population-based study. Neuroepidemiology. 2019;52:119-27.

13 Agency for Healthcare Research and Quality. Healthcare Cost and Utilization Project (HCUP)-US National (Nationwide) Inpatient Sample (NIS) Overview. Available from: https: //www.hcup-us.ahrq.gov/nisoverview.jsp.

14 NHS Digital: Hospital Episode Statistics (HES). Available from: https://digital.nhs.uk/ data-and-information/data-tools-and-services/data-services/hospital-episode-statistics.
15 République Française Ministère des Solidarités et de la Santé: Direction de la recherche, des études, de l'évaluation et des statistiques (DREES). Available from: http://www.data. drees.sante.gouv.fr/ReportFolders/reportFolders.aspx?sCS_referer $=\&$ sCS_ChosenLang=fr.

16 Tuckuviene R, Christensen AL, Helgestad J, Johnsen SP, Kristensen SR. Paediatric arterial ischaemic stroke and cerebral sinovenous thrombosis in Denmark 1994-2006: a nationwide population-based study. Acta Paediatr. 2011;100:543-9.

17 Gesundheitsberichterstattung des Bundes: The Federal Health Monitoring System. Available from: http://www.gbe-bund.de/ gbe10/pkg_navigation.prc_sitemap?p_ uid=gastg\&p_aid=0\&p_sprache $=E \& p \_h e l p \_$ context=H_HOME.

18 Gobierno de España Ministerio de Sanidad: Conjunto Mínimo Básico de Datos - Hospitalización (CMBD-H): Diagnósticos Hospitalización (incluye Sector Privado). Available from: https://pestadistico.inteligenciadegestion.mscbs.es/publicoSNS/N/rae-cmbd/ cmbd-h/diagnosticos-hospitalizacion-incluye-sector-privado.

19 Stacey A, Toolis C, Ganesan V. Rates and risk factors for arterial ischemic stroke recurrence in children. Stroke. 2018;49:842-7.

20 Fullerton HJ, Wintermark M, Hills NK, Dowling MM, Tan M, Rafay MF, et al. Risk of recurrent arterial ischemic stroke in childhood: a prospective international study. Stroke. 2016;47:53-9. 
21 Fullerton HJ, Wu YW, Sidney S, Johnston SC. Risk of recurrent childhood arterial ischemic stroke in a population-based cohort: the importance of cerebrovascular imaging. Pediatrics. 2007;119:495-501.

22 StatsDirect Limited: Poisson Rate Confidence Interval 2020. Available from: https://www. statsdirect.com/help/rates/poisson_rate_ ci.htm.

23 Neyeloff JL, Fuchs SC, Moreira LB. Metaanalyses and Forest plots using a Microsoft Excel spreadsheet: step-by-step guide focusing on descriptive data analysis. BMC Res Notes. 2012;5:52.

24 Grunt S, Mazenauer L, Buerki SE, Boltshauser E, Mori AC, Datta AN, et al. Incidence and outcomes of symptomatic neonatal arterial ischemic stroke. Pediatrics. 2015;135:e12208.

25 Giroud M, Lemesle M, Gouyon JB, Nivelon JL, Milan C, Dumas R. Cerebrovascular disease in children under 16 years of age in the city of Dijon, France: a study of incidence and clinical features from 1985 to 1993 . J Clin Epidemiol. 1995;48:1343-8.

26 Earley CJ, Kittner SJ, Feeser BR, Gardner J, Epstein A, Wozniak MA, et al. Stroke in children and sickle-cell disease: Baltimore-Washington Cooperative Young Stroke Study. Neurology. 1998;51:169-76.
27 Mallick AA, O'Callaghan FJ. Prospective studies of the incidence of pediatric arterial ischaemic stroke. Blood Cells Mol Dis. 2018; 69:101.

28 Munoz D, Hidalgo MJ, Balut F, Troncoso M, Lara S, Barrios A, et al. Risk factors for perinatal arterial ischemic stroke: a case-control study. Cell Med. 2018;10:2155179018785341.

29 Lee J, Croen LA, Backstrand KH, Yoshida CK, Henning LH, Lindan C, et al. Maternal and infant characteristics associated with perinatal arterial stroke in the infant. Jama. 2005; 293:723-9.

30 Ferriero DM, Fullerton HJ, Bernard TJ, Billinghurst L, Daniels SR, DeBaun MR, et al. Management of stroke in neonates and children: a scientific statement from the American Heart Association/American Stroke Association. Stroke. 2019;50:e51-96.

31 Rambaud T, Legris N, Bejot Y, Bellesme C, Lapergue B, Jouvent E, et al. Acute ischemic stroke in adolescents. Neurology. 2020;94: e158-69.

32 Tsze DS, Valente JH. Pediatric stroke: a review. Emerg Med Int. 2011;2011:734506.

33 Hills NK, Johnston SC, Sidney S, Zielinski BA, Fullerton HJ. Recent trauma and acute infection as risk factors for childhood arterial ischemic stroke. Ann Neurol. 2012;72:850-8.
34 Mortazavi MM, Verma K, Tubbs RS, Harrigan M. Pediatric traumatic carotid, vertebral and cerebral artery dissections: a review. Childs Nerv Syst. 2011;27:2045-56.

35 Jackson SL, Zhang Z, Wiltz JL, Loustalot F, Ritchey MD, Goodman AB, et al. Hypertension among youths - United States, 20012016. MMWR Morb Mortal Wkly Rep. 2018; 67:758-62.

36 George MG, Tong X, Kuklina EV, Labarthe DR. Trends in stroke hospitalizations and associated risk factors among children and young adults, 1995-2008. Ann Neurol. 2011; 70:713-21.

37 Kupferman JC, Zafeiriou DI, Lande MB, Kirkham FJ, Pavlakis SG. Stroke and hypertension in children and adolescents. J Child Neurol. 2017;32:408-17.

38 Kim JS. Moyamoya disease: epidemiology, clinical features, and diagnosis. J Stroke. 2016; 18(1):2-11.

39 Sato Y, Kazumata K, Nakatani E, Houkin K, Kanatani Y. Characteristics of moyamoya disease based on national registry data in Japan. Stroke. 2019;50:1973-80.

40 The World Bank: World Bank country and lending groups. Available from: https://datahelpdesk.worldbank.org/knowledgebase/ articles/906519\#High_income. 